\title{
A prospective audit of pacemaker function, implant lifetime, and cause of death in the patient
}

\author{
S K Suvarna, R D Start, D I Tayler
}

\begin{abstract}
Aim-To audit prospectively the reasons for pacemaker implantation, the duration of the pacemaker use, the cause of death, and pacemaker function after removal from the patient.

Methods-Pacemakers were removed at necropsy, or from the bodies of patients awaiting cremation, in three hospitals over a three year period. The cause of death was taken from the results of the necropsy or from the certified cause of death. Demographic data, including the time of implant and reasons for implantation, were checked. The pacemakers were analysed in terms of battery status, program, and output under a standard 470 ohm load.
\end{abstract}

Results-69 patients were studied. Average age at death was 78 and 80 years for men and women, respectively. The average duration since pacemaker implantation was 46 months. Eleven patients had necropsies, showing that three died from ischaemic heart disease, six from cardiomyopathy, one from an aortic aneurysm, and one from disseminated neoplasia. From the necropsy results and death certificates, the distribution of causes of death in the group as a whole were ischaemic heart disease (21), cardiomyopathy (8), cerebrovascular disease (11), neoplasia (11), chest infection/chronic obstructive airways disease (8), and other causes (10). In all cases the pacemaker box function was within normal limits.

Conclusions-Neither primary nor secondary pacemaker dysfunction was found. The study highlights the impact of arrhythmias in cardiomyopathy, and raises questions about the true role of ischaemic heart disease in these pacemaker requiring patients. The relatively short gap between pacemaker implantation and death requires further study.

(f Clin Pathol 1999;52:677-680)

Keywords: pacemaker; arrhythmias; mortality

The modern cardiac pacemaker is a commonly employed device used for the control of various arrhythmias. ${ }^{1}$ It has a good record of safety and efficacy. Recently models capable of delivering dc shocks directly to the myocardium to terminate life threatening tachyarrythmias have been introduced. ${ }^{2}$ While there are some published data on the reasons for pacemaker implantation, ${ }^{3}$ the causes of death in patients with pacemakers, ${ }^{4}$ and pacemaker assessment post mortem, ${ }^{5-7}$ our aim in this study was to make a prospective audit of pacemaker function after death in patients from our cardiology caseload and to cross correlate this with the cause of death and other demographic data.

\section{Methods}

Over three years, at three hospitals, two pathologists collected cardiac pacemakers from deceased patients awaiting cremation and from cases on which a necropsy had been held. The connections and lead implantation sites were checked where possible, and the explanted units were sent for formal electronic testing. This involved a check on battery status and performance under a standard $470 \mathrm{ohm}$ load. Program details were checked by electronic interrogation of the pacemaker unit according to the manufacturer's instructions.

The patient's demographic details (age at death, date of birth, age at pacemaker implantation) were recorded. The certified cause of death was checked with the patient's record (where available) and in those cases having a necropsy the established cause of death was recorded. The reasons for pacemaker implantation were also audited. Pacemakers in deceased patients awaiting burial were not available for assessment.

Results

In all, 69 patients and pacemakers were recruited into the study (31 men and 31 women). The distribution of their ages at death is given in fig 1. Age at death was skewed towards the elderly end of the spectrum. The

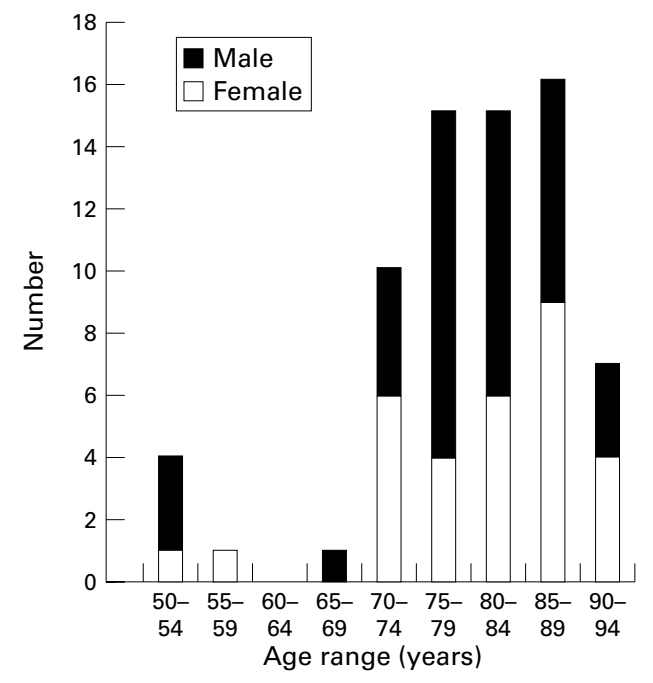

Figure 1 Bar histogram of the patient's age at death versus case frequency. 


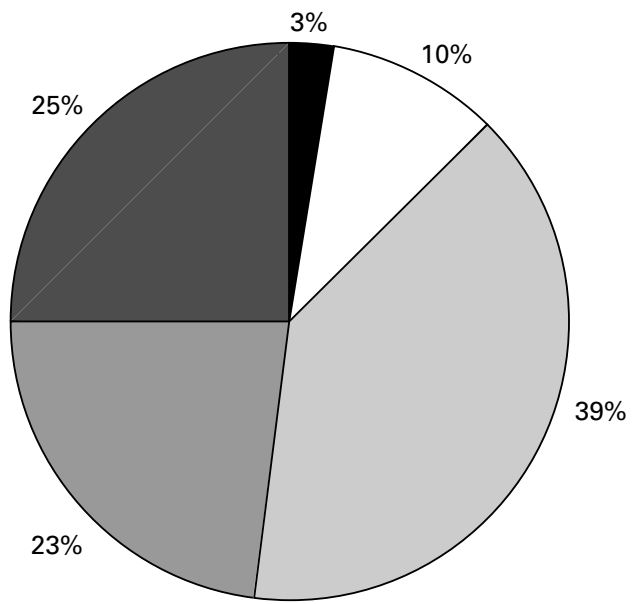

\begin{tabular}{|l}
\hline 1st degree HB \\
$\square$ 2nd degree HB \\
$\square$ 3rd degree HB \\
$\square$ sss \\
$\square$ Arrhythmia/NOS
\end{tabular}

Figure 2 Pie chart showing the indications for pacemaker implantation in the patients studied. NOS, .

gap between male and female age at death was small (men 78.2 years, women 80.5 years), the average being 79.2 years.

The reasons for pacemaker implantation parallel the results of other studies ${ }^{3}$ (fig 2) The study revealed 27 cases of third degree heart block, seven of second degree block, and two with first degree block. Sick sinus syndrome was present in 16 patients. There were, however, 17 cases in which "arrhythmia NOS" (not otherwise specified) and/or "syncopal attacks NOS" were given as the indications for unit implantation.

The causes of death in the group as a whole (from death certificates and necropsy findings) were ischaemic heart disease (21), cardiomyopathy (8), cerebral vascular disease (11), neoplasia (11), chest infection and/or chronic obstructive airways disease (8), and other causes, including peripheral vascular disease, renal failure, gastrointestinal haemorrhage, and so on (10). These data are displayed graphically in fig 3 . In the cases coming to necropsy, six had cardiomyopathy, three had ischaemic heart disease, one had an abdominal aortic aneurysm, and one had disseminated neoplasia. In these latter two cases, the underlying cardiac lesion was coronary ischaemia. The cases with cardiomyopathy had amyloid heart disease (2), dilated cardiomyopathy NOS (2), hypertrophic cardiomyopathy (1), and cardiomyopathy associated with systemic lupus erythematosus (1). The five cases in which death occurred below the age of 60 years included three with cardiomyopathy and two with ischaemic heart disease.

All the pacemakers functioned within normal limits when tested following explantation. Time since implantation ranged between one day and 11 years. The average lifetime of the implant was 46 months. One case involving a
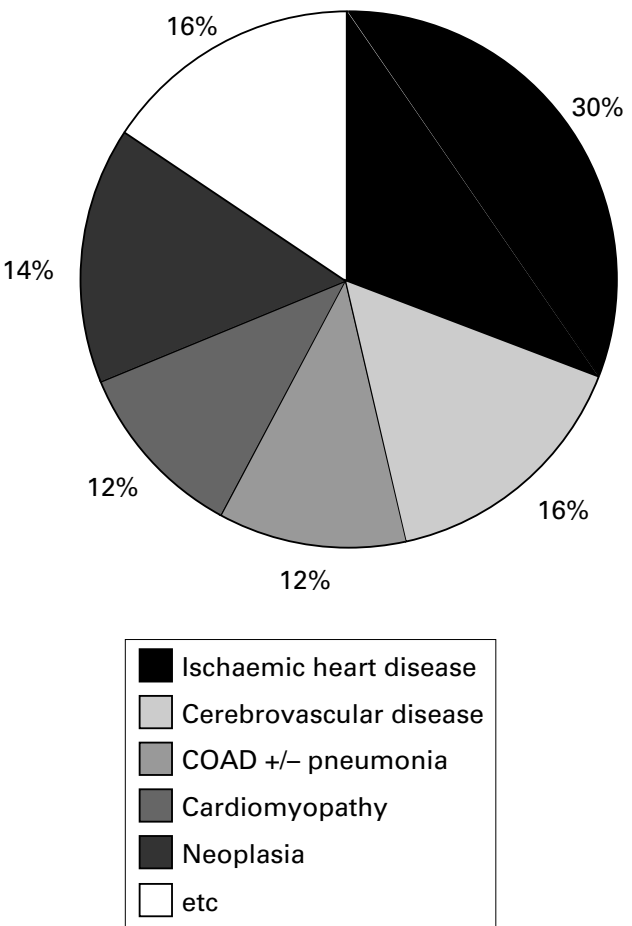

Figure 3 Pie chart showing the causes of death (from death certificates and necropsy findings) in the group as a whole.

defibrillator pacemaker unit (fig 4) was encountered. This unit was removed before it became evident that the defibrillator had not been deactivated.

\section{Discussion}

Pacemakers are common devices employed for the management of a variety of arrhythmias. ${ }^{1}$ Following their introduction, ${ }^{89}$ over the last 40 years their design has improved and relatively few suffer failure, either primary (program failure, lead fracture) or secondary (battery depletion, "twiddler" damage). ${ }^{10}$ In this study we set out to audit pacemaker function in three hospitals by harvesting the pacemaker units from deceased patients, recording their demographic data, and formally testing the explanted units electronically.

Although the number of patients recruited into the study is relatively small, the reasons for pacemaker implantation reflect those in other studies, ${ }^{3}$ and we are confident that we were not dealing with a biased cohort.

One of the prime objectives for the study was the assessment of pacemaker status after death. We were reassured to find that all the units studied had good outputs against a standard $470 \mathrm{ohm}$ load, with adequate battery reserve and functioning programs. Within the necropsy subgroup, the ability to inspect the entirety of the system directly, from pacemaker box along the wire to the implantation site, was employed to exclude wire fracture, implantation misplacement, and infective endocarditis. None of these features was identified, although some non-specific thrombus and minor fibrous adhesions to atrial tissues were noted. ${ }^{11}$

The benefit of this thorough testing was particularly relevant in those cases that came to 


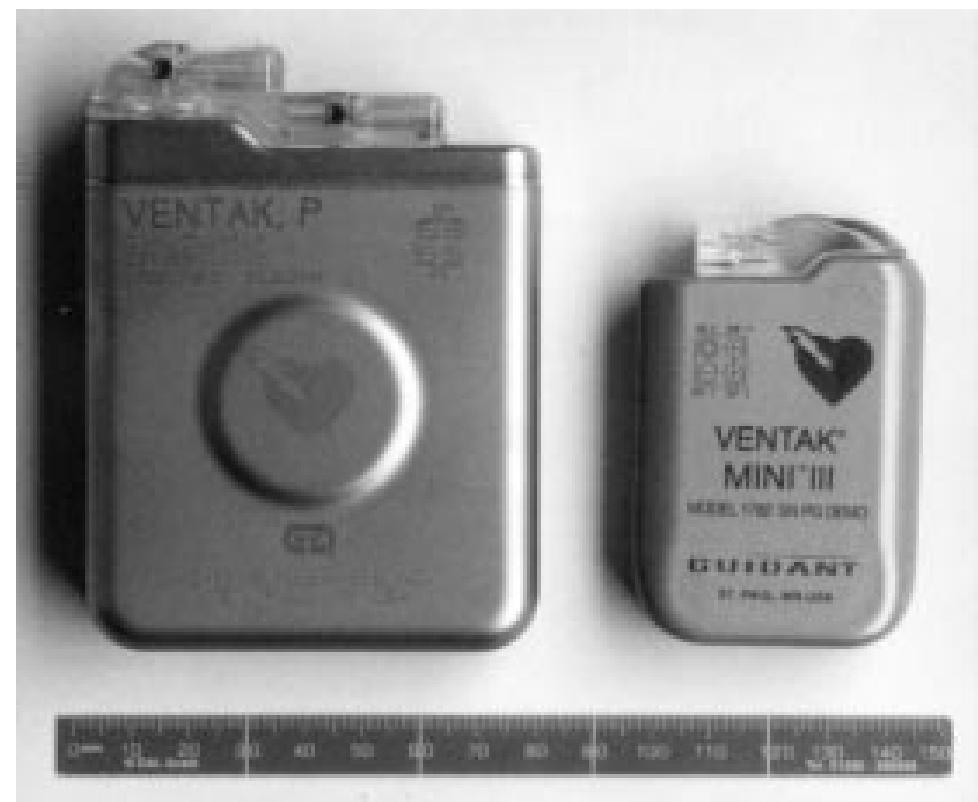

Figure 4 A defibrillator pacemaker unit showing the "lightning flash" symbol.

coroner's necropsy. These examinations, often carried out in cases of sudden death, might conceivably involve pacemaker dysfunction, so electronic unit interrogation is pertinent in medico-legal necropsies of fatalities involving road accidents and other forms of accidental death. ${ }^{5}$

Pacemaker unit assessment within the pacemaker clinic had the additional benefit that we were able to remove some deceased patients from the routine clinic recall programme. This avoided the unfortunate situation where deceased patients were sent clinic appointments because of failure to update the database. To augment the recovery of deceased patients' pacemaker units we have issued local hospitals with proformas encouraging those removing pacemakers to return them intact to our centre with details of the cause of death.

The safe disposal of pacemakers is important. While it is acceptable for pacemakers to be left in situ in patients awaiting burial, there is a specific requirement for the removal of pacemakers if cremation is to take place, to prevent the possibility of explosion. By requesting the return of pacemakers to our centre we also hope to avoid the inadvertent placement of a pacemaker in a standard incineration box of hospital waste.

The defibrillator pacemaker is being used increasingly, ${ }^{2}$ and we were not surprised that one case featured within our cohort. There is a suggestion that malfunction in these units is associated with increased mortality. ${ }^{12}$ Such units are capable of discharging a 30 joule shock and should ideally be deactivated before any manipulation is undertaken, as recommended in patients undergoing surgery. ${ }^{13}$ Accidental discharge through scissors cutting the defibrillator leads could be hazardous to medical or mortuary staff. ${ }^{14}$ It is helpful that defibrillator pacemakers bear a hazard warning "lightning flash" logo (fig 4).
The certificated causes of death showed a bias towards cases of ischaemic heart disease. If one accepts the limitations of certificated cause of death, ${ }^{15}$ then extrapolating from our necropsy caseload (in which six of the 11 cases had an underlying cardiomyopathy), it is possible that cardiomyopathy features more largely in a pacemaker requiring population than hitherto suggested. The small numbers in our study preclude any further interpretation, but future data collection should provide greater insight into the causes of arrhythmias.

The modern pacemaker has a battery life of five to 10 years, depending on use. The finding that the average time between implantation and death was 46 months was also notable. No pattern was discerned in those with shorter "lifetimes," but there is scope for further work in this area as pacemakers and the patient care required following implantation are relatively expensive. ${ }^{16}$ There has been some work examining patient longevity following pacemaker implantation in the very elderly. ${ }^{17}$ Given the availability of dual lead, multiprogram models alongside standard VVI pacemakers it would be financially sensible to target pacemaker models at specific patient populations. ${ }^{17}$ Also, as some of the pacemakers have short implantation times, it might be argued that we should reuse the explanted units, as is the practice in some other countries. ${ }^{18}{ }^{19}$ However, at present those units retrieved and deemed suitable are only available for appropriate veterinary purposes from our centre.

In conclusion, this study confirms the integrity of pacemaker units in patients dying in and around Sheffield and shows no evidence of secondary pacemaker failure. The study highlights the impact of cardiomyopathy in the population requiring a pacemaker, and raises questions as to the real impact of ischaemic heart disease.

We are grateful to Mrs Margaret Peach and Mrs Denise Dunbar, senior cardiology technicians, Northern General Hospital, Mr Michael Rigby, superintendent registrar, Sheffield Registry, and Mrs Seonaid Ashby, secretary, for their assistance with this study and the preparation of the manuscript.

1 Working Party of the British Pacing and Electrophysiology Group. Recommendations for pacemaker prescription for symptomatic bradycardia. Br Heart $\mathcal{F} 1991$;66:185-9

2 Teo KK. Recent advances. Cardiology. BMF 1998;316:91115

3 Goldman BS, Nishimura S, Lau C. Survey of cardiac pacing in Canada (1993). Can J Cardiol 1996;12:573-8.

4 Mattioli AV, Rossi R, Annicchiarico E, et al. Causes of death in patients with unipolar single chamber ventricular pacing: prevalence and circumstances in dependence on arrhythmias leading to pacemaker implantation. PACE 1995;18: $11-17$.

5 Haga $\mathrm{K}$, Terazawa $\mathrm{K}$, Ishimori $\mathrm{N}$, et al. An autopsy case of a pacemaker implanted patient. $\mathcal{F p}_{p} \mathcal{F}$ Legal Med 1995;49: pacem 8 .

6 Raasch F. Pacemaker postmortem. In: Legal medicine annual. Boston: Butterworth, 1997:97-110.

7 Cox JN. Pathology of cardiac pacemakers and central catheters. In: Berry CL, ed. The pathology of devices. Berlin: Springer-Verlag, 1993

8 Furman S, Schwedel JB. Transvenous pacing. $N$ Engl f Med 1959;261:943-8.

9 Chardack WM, Gage AA, Greatbatch W, et al. Surgery 1960;48:643-54.

10 Hayes DL, Vlietstra RE. Pacemaker malfunction. Ann Intern Med 1993;119:828-35.

11 Robboy SJ, Harthorne JW, Leinbach RC, et al. Autopsy findings in permanent pervenous pacemakers. Circulation 1969;39:495-501.

12 Pfeiffer D, Jung W, Fehske W, et al. Complications of pacemaker-defibrillator devices: diagnosis and management. Am Heart F 1994;127:1073-80. 
13 Bourke ME. The patient with a pacemaker or related device. Can F Anaesth 1996;43:R24-41.

14 Prahlow JA, Guileyardo JM, Barnard JJ. The implantable cardioverter-defibrillator. A potential hazard for autopsy pathologists. Arch Pathol Lab Med 1997;121:1076-80.

15 Royal College of Pathologists. The autopsy and audit. London: The Royal Colleges of Pathologists, Physicians and Surgeons, 199 16 Mounsey JP, Ray SG, Griffith MJ, et al. Impact of internal
audit on pacemaker prescription and the immediate costs of pacing in the Northern Region: towards implementation of the recommendations of the British Pacing and Electrophysiology Group. Br Heart $\mathcal{F}$ 1994;71:395-8.

Shen WK, Hayes DL, Hammill SC, et al. Survival and functional independence after implantation of a permanent pacemaker in octogenarians and nonagenarians. Ann Intern Med 1996;125:476-80.

8 Panja M, Sarkar CN, Kumar S, et al. Reuse of pacemaker. Indian Heart $\mathcal{F}$ 1996;48:677-80.

19 Sethi KK, Bhargava M, Pandit N, et al. Experience with recycled cardiac pacemakers. Indian Heart $f$ 1992;44:91-3. 